\title{
Digital Image Restoration with Nonlinear Smoothing for Circular Motion Blurred Images
}

\author{
W. Fawwaz Al Maki, T. Hori, T. Kitagawa, and S. Sugimoto \\ Dept. of Electrical and Electronic Engineering, Ritsumeikan University \\ 1-1-1 Noji-Higashi, Kusatsu City, Shiga 525-8577, Japan \\ E-mail: sugimoto@se.ritsumei.ac.jp
}

\begin{abstract}
In this paper, we present an image restoration technique for noiseless circular motion blurred images. To simulate the circular motion blurred images, we create circular paths by applying the Bresenham line algorithm. The algorithm can simplify the spatially variant image restoration problem into the spatially invariant one. Since the degradation process along the circular blurring paths is spatially invariant, the sharp images can be obtained by applying a direct inverse filtering method in the spatial domain. Here, inverse filtering is applied to each circular path. Finally, we apply the nonlinear smoothing techniques to enhance the restored images. Experimental results will show the feasibility of the presented blurred image discretization and restoration method.
\end{abstract}

\section{Introduction}

Circular motion blurred images are sometimes unavoidable in a digital camera operation, industry, or medical imaging system apparatus. Obtaining the sharp images from such blurred images by using image restoration procedure is an interesting problem. As the circular motion blur is spatially variant, the corresponding degraded image can not be restored by using image restoration algorithms which do not deal with the spatially variant point spread function. Thus, restoring the spatially variant blurred image is a difficult problem.

Generally, the spatially variant blurred image restoration method can be divided into two groups: direct restoration method and indirect restoration method. In the direct method, the blurred image is restored directly by considering a spatially variant point spread function whereas in the indirect method, the spatially variant restoration problem is reduced into the spatially invariant one by using a decomposition technique. Many researchers have proposed methods to directly restore the spatially variant blurred image $[1,2,3]$. In [3], the blurred image is restored by using a variational method that estimate simultaneously sharp image and depth map, based on the minimization of a cost functional. However, the author considered the special case, i.e. the camera moves in one plane perpendicular to the optical axis without any rotations.

In the circular motion blurred image restoration problem, it is important to simulate the blurred image. Since restoration of the real circular motion blurred image needs the point spread function (PSF) parameters estimation, the spatially variant restoration problem in the circular motion blurred image can not be solved without the knowledge of the PSF parameters. This problem is called the blind image restoration problem. In this paper, we simulate the blurred image to verify the reliability and feasibility of our method. Also, in the blind image restoration problem, the simulated blurred image can be used to demonstrate accuracy of the parameters estiomation method. Then, the estimation and restoration method can be applied to the real motion blurred images.

To simulate a circular motion blurred image, it is important to prepare circular paths as the blurring paths. The log-polar sampling $[4,5,6,7]$ or foveated mapping can be used to generate the blurring paths. In the log-polar mapping, the rectangular coordinate system of image plane $(x, y)$ is transformed to the log-polar coordinate system $(\zeta, \eta)$ where $\zeta=\log \sqrt{(x+a)^{2}+y^{2}}$ and $\eta=\arctan \frac{y}{x+\alpha}$.

A geometric coordinate transformation that transforms the rectangular lattice of image plane to polar lattice can also be used to generate the blurring paths $[8,9,10]$. In this method, a mapping rule between pixels in the rectangular and polar coordinate systems is needed to transform a coordinate system to another coordinate system. Transformation from the rectangular coordinate system to the polar coordinate system needs sampling in both radial and rotational directions. Sampling interval for both directions must be determined 
carefully.

In the geometric coordinate transformation technique, sampling in the polar coordinate system is an important point since it leads to a trade off between high computation problem and undersampling. The more samples in the polar coordinate system are, the higher the computation time is needed. In this condition, the blurred image can be more perfectly simulated. Since there are more samples in the polar coordinate system, the blurred image can be restored more succesfully. The opposite condition will happen if there are less samples in the polar coordinate system.

However, all methods have evident drawbacks, i.e. sampling resolution and high computational time since they involve trigonometric and/or logarithmic operations. The high computational time becomes a more serious problem in the real-time applications. A reliable method to simulate the circular motion blurred images is needed. Here, we use a circle generating algorithm that adapts the Bresenham's line algorithm [11]. In generating the circular paths, the Bresenham algorithm only involves incremental integer calculations. The algorithm also gives significant contributions to the image restoration since the use of it can alleviate us in displaying the results of image restoration in the rectangular coordinate system of image plane. Here, we restore the blurred image in the polar coordinate system.

In this paper, spatially variant restoration problem for the circular motion blurred image is considered. To alleviate the restoration of circular motion blurred image, the spatially variant model is reduced into the spatially invariant one by modeling the circular motion blurred image along the circular path. The circular motion blur in each circular path is analogous to the uniform linear motion blur. Thus, the spatially invariant restoration technique for the uniform linear motion blur can be applied to remove the circular motion blur in each circular path. Here, we emphasize on the method to simulate the circular motion blurred image and an image restoration algorithm to remove circular motion blur from an image. Therefore, in this paper, parameter estimation is not incorporated since the circular motion blur parameter, i.e. center of rotation and angle of rotation or blur length, are given.

\section{Circular Motion Blurred Images}

\subsection{Image Degradation Analysis}

Although a circular motion blurred image is spatially varying in the rectangular coordinate system, it should be expressed as an output of spatially invariant system to alleviate the restoration. To achieve this, the blurred image is expressed in the polar coordinate system. For the constant angular velocity case, it is defined as

$$
\begin{aligned}
z(r, \theta) & =\frac{1}{t_{\exp }} \int_{0}^{t_{\exp }} u(r, \theta-\omega t) \mathrm{d} t \\
& \cong \frac{1}{t_{\exp } k \Delta} \sum_{j=0}^{(k-1) \Delta T} u(r, \theta-j \omega \Delta T)
\end{aligned}
$$

For each $r$, by using $\alpha=\theta-\omega t$, (1) can be expressed as

$$
z_{r}(\theta)=\frac{1}{T} \int_{\theta-\omega T}^{\theta} u_{r}(\alpha) \frac{1}{\omega} \mathrm{d} \alpha ; \quad T=t_{\exp }
$$

As the circular motion blur is spatially invariant in the polar coordinate form, it becomes similar to a linear motion blur that occurs along the circular paths. The point spread function of the circular motion blur along the circular paths is given by

$$
h(s)= \begin{cases}\frac{1}{\omega T} & \text { for } 0 \leq s \leq \omega T \\ 0 & \text { otherwise }\end{cases}
$$

where $\omega$ is the angular velocity. The term $\omega T$ in (4) is the blur length along the circular paths. In the circular rotation motion blurred image case, the blur length is basically an angle of rotation that caused degradation on an image.

\subsection{Method for Simulating the Blurred Image}

To simulate circular motion blurred images, the blurring paths in the polar coordinate system must be perpared. Rather than using coordinate system mapping technique and interpolation method, we use a circle generating algorithm that adapts the Bresenham line algorithm to generate a series of circular paths as the motion blurring paths. In this algorithm, a circle function is used [11]:

$$
f_{\text {circle }}(x, y)=x^{2}+y^{2}-r^{2}
$$

Any point $(x, y)$ on the boundary of the circle with radius $r$ satisfies the equation $f_{\text {circle }}(x, y)=0$. If the point is in the interior of the circle, the circle function is negative. If the point is outside of the circle, the circle funstion is positive. Thus, the relative position of any point $(x, y)$ can be determined by checking the sign of the circle function. The circle function is applied to midpoints between pixels near the circle path at each sampling step [11]. By using the midpoint, pixels along the circumference of a circle can be obtained by using integer operations. 
We assume that center of the circle is at the origin point $(0,0)$ and $k_{r}$ is the discrete coordinates index for radius $r$. Then, the initial value of decision parameter is obtained by evaluating the circle function at position $P_{0} \equiv\left(x_{0}, y_{0}\right)=\left(0, k_{r}\right)$, and is given by [11]

$$
D_{0}=f_{\text {circle }}\left(1, k_{r}-\frac{1}{2}\right)=1+\left(k_{r}-\frac{1}{2}\right)^{2}-k_{r}^{2},
$$

where $k_{r}=1,2, \cdots, K_{r}$. The decision paramater $D$ is calculated successively to determine the closer pixel to the circular path. For each $x_{k}$, starting at $k=0$, the next point $\left(P_{k+1}\right)$ and the next decision parameter $\left(D_{k+1}\right)$ are given by

$$
\begin{gathered}
P_{k+1}= \begin{cases}\left(x_{k+1}, y_{k}\right) & \text { if } D_{k}<0, \\
\left(x_{k+1}, y_{k}-1\right) & \text { if } D_{k} \geq 0\end{cases} \\
D_{k+1}= \begin{cases}D_{k+1}=D_{k}+2 x_{k}+3 & \text { if } D_{k}<0, \\
D_{k+1}=D_{k}+2 x_{k}+5-2 y_{k} & \text { if } D_{k} \geq 0\end{cases}
\end{gathered}
$$

The degradation process along the circular path, i.e. at $k_{r}$-th radius, is given by

$$
z_{k_{r}}(n)=\sum_{l=0}^{L-1} \frac{1}{L} u_{k_{r}}(n-l)
$$

where $n=1,2, \cdots, N$. By applying the circle generation algorithm, we obtain a new matrix with size $K_{r} \times N$. From (9), it is evident that the circular motion blurring process along the circular path is analogous to the uniform linear motion blurring process.

In (9), $L$, which is the blur length, is a number of pixels along the circular path that contribute to the blurring process. For a given rotation angle $\theta$, it is obtained by using

$$
L=\left\lceil\frac{\theta}{360} 2 \pi k_{r}\right\rceil
$$

The aforesaid formula shows that angle of rotation has a relation with the blur length. However, for the same rotation angle, the blur length will be different depending on the radius of the circular blurring path. In other words, the number of image scene points that affects an image pixel depends on the distance of the image pixel from the center of rotation.

Since the Bresenham line algorithm works on the digital image, some pixel position can not be fetched along the circular paths. This leads to empty pixels problem. Linear interpolation is difficult to apply to estimate the empty pixels. To perform linear interpolation, we need 4 pixels to estimate an empty pixel. However, many empty pixels are located very close one to each other.
Therefore, the pixels needed to estimate an empty pixel are often the empty pixels as well.

To overcome the empty pixels distortion problem, we used nonlinear smoothing. By using nonlinear smoothing, the image structure can be kept intact. Also, the nonlinear smoothing method will not reduce the quality of whole image. Here, we use median filtering to estimate the blurred image pixels at location of the empty pixels. The median filter replaces every image pixel by the median of the pixels in the corresponding filter region $R$ [12]:

$$
U^{\prime}(m, n) \leftarrow \operatorname{median}\{U(m+i, n+j) \mid(i, j) \in R\}
$$

The median of $2 K+1$ pixel values $p$ is defined as

$$
p_{K} \equiv \operatorname{median} \cdot\left(p_{0}, p_{1}, \ldots, p_{K}, \ldots, p_{2 K}\right) ;
$$

i.e. the center value $p_{K}$ if the sequences $\left(p_{0}, \ldots, p_{2 K}\right)$ is sorted $\left(p_{i} \leq p_{i+1}\right)$.

\section{Image Restoration}

The image restoration method presented in this paper is based on the assumption of spatially invariant degradation process along the circular blurring path. If the spatially invariant degradation process in (9) is expressed in a matrix form, we get

$$
\mathbf{z}_{k_{r}}=\mathbf{H} \mathbf{u}_{k_{r}}
$$

where $\mathbf{z}_{k_{r}} \in \Re^{N \times 1}, \mathrm{H} \in \Re^{N \times N}$, and $\mathbf{u}_{k_{r}} \in \Re^{N \times 1}$ are defined by the following matrices

$$
\begin{aligned}
& \mathbf{z}_{k_{r}}=\left[\begin{array}{c}
z_{k_{r}}(1) \\
z_{k_{r}}(2) \\
\vdots \\
\vdots \\
z_{k_{r}}(N)
\end{array}\right] \quad \mathbf{u}_{k_{r}}=\left[\begin{array}{c}
u_{k_{r}}(1) \\
u_{k_{r}}(2) \\
\vdots \\
\vdots \\
u_{k_{r}}(N)
\end{array}\right] \\
& \mathbf{H}=\frac{1}{L}\left[\begin{array}{cccccc}
1 & & & & & \\
\vdots & \ddots & & & 0 & \\
\vdots & \ddots & \ddots & & & \\
1 & \ddots & \ddots & \ddots & & \\
& \ddots & \ddots & \ddots & \ddots & \\
0 & & 1 & \ldots & \ldots & 1
\end{array}\right]
\end{aligned}
$$

In (14), elements of $\mathbf{u}_{k_{r}}$ and $\mathbf{z}_{k_{r}}$ are pixels of the original image and the blurred image, respectively, along the $k_{r}$-th circular path. From (13), the restored image can be obtained by employing inverse filtering along the blurring path. Since the degradation process of the 
circular motion along blurring path in the polar coordinate system is similar to that of the linear motion blur, the method presented in [13] can be used here. Therefore, the restoration of circular motion blurred image is realized by applying the folowing equation to each radius $\left(k_{r}=1,2, \ldots K_{r}\right)$.

$$
\hat{\mathbf{u}}_{k_{r}}=\mathbf{H}^{-1} \mathbf{z}_{k_{r}}
$$

However, since the inverse filter is applied to the blurred image pixels along the circular path and not all image pixels can be fetched to the circular path by the Bresenham algorithm, some unrestored image pixels will remain. To overcome this problem, again nonlinear smoothing technique, i.e. median filtering, is applied to the restored image to obtain a complete restored image.

\section{Experimental Results and Discussion}

We applied the Bresenham line algorithm to the monochrome images. However, the algorithm can also be applied to color images (RGB images). In the color image case, to simulate a circular motion blurred image, the Bresenham algorithm was applied to only one color channel to calculate the pixel positions along the circular paths. Then, the blurring process was applied to every color channel. If we use the geometric coordination transformation method, it is important to perform interpolation and coordinate transformation for each color channel, not only the blurring process. This condition will burden the computation.

Fig. 1 shows an original monochrome image. Fig. 2 shows the circular motion blurred image simulated by using the Bresenham algorithm. However, since some image pixels are located beyond the circular paths, blurring process can not be simulated to whole image pixels. As a result, the image shown in Fig 2 is not fully blurred. Fig. 3 shows the circular motion blurred image after applying median filtering to Fig. 2.

In the image restoration, direct inverse filter is applied to each circular pathof the blurred image. Fig. 4 shows the restored image. However, since the inverse filter was not applied to the whole image pixels, some unrestored image pixels existed in the image. The unrestored image pixels were located beyond the circular path. After applying nonlinear smoothing, i.e. median filtering, the sharp image could be obtained as shown in Fig. 5. Fig. 5 shows that the restoration method with median filtering can remove the motion blur from the image completely.

To show the effect of restoration, we also calculated MSE (mean square error) for several standard images.

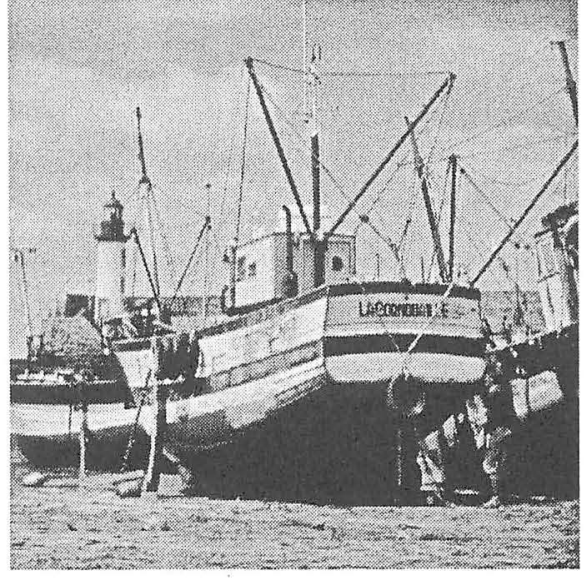

Fig. 1: Original Image

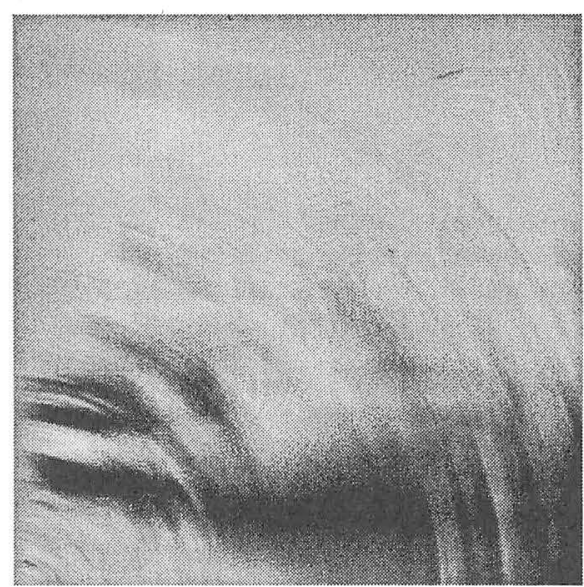

Fig. 2: Simulated Blurred Image before Nonlinear Smoothing

MSE can express the relation between the restored image and the original image. MSE is expressed as

$$
M S E=\frac{1}{M N} \sum_{m=0}^{M-1} \sum_{n=0}^{N-1}[\hat{U}(m, n)-U(m, n)]^{2}
$$

where $\hat{U}(m, n)$ and $U(m, n)$ denote the restored image and the original image, respectively. Table. 1 shows the MSE values for several standard images.

We also show the influence of median filtering. Here, we calculated two kinds of MSE, i.e. the MSE calculated before and after nonlinear smoothing was applied to the restored image. Table. 1 shows that median filtering can reduce the MSE. Therefore, median filtering proves to be effective to solve the empty pixels distortion in the restored image. This fact can also be seen in the Fig. 5.

From Table. 1, we obtain that the edges in an image will give influence to the calculated MSE, especially 


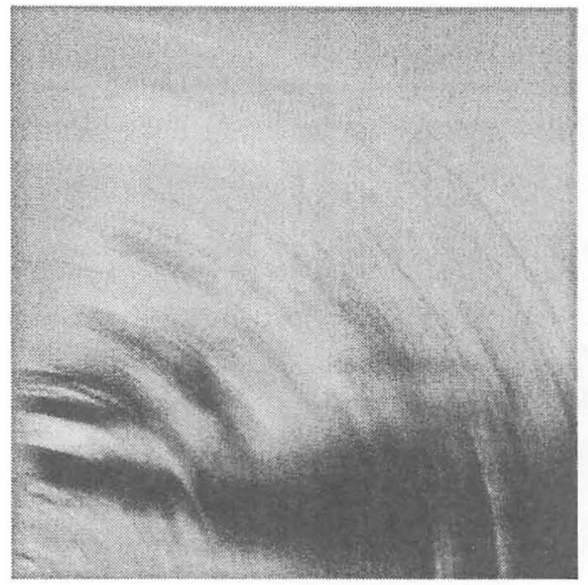

Fig. 3: Simulated Blurred Image after Nonlinear Smoothing

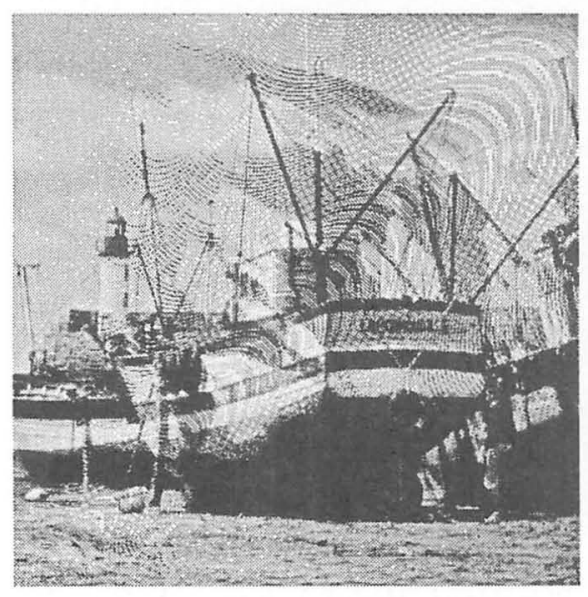

Fig. 4: Restored Image without Nonlinear Smoothing

$\mathrm{MSE}_{\mathrm{as}}$. Compared to the other standard images used in this paper, the boat image gets comparatively small effect of median filtering. However, the final restored image shown in Fig. 5 is close to Fig. 1.

In Table. 1, the Girl image has the lowest $\mathrm{MSE}_{\mathrm{as}}$. This image has less edges than the Boat image does. In our simulation, the image gives the best result. Fig. 6, Fig. 7, and Fig. 8 show the original image, the blurred image, and the restored image, respectively.

From our experimental results, it is evident that Bresenham line algorithm with nonlinear smoothing can simulate the circular motion blurred image. The algorithm is also useful in the image restoration. For the given center of rotation and the blur length or angle of rotation, the combination of the algorithm and the nonlinear smoothing can restore teh blurred image succesfully.

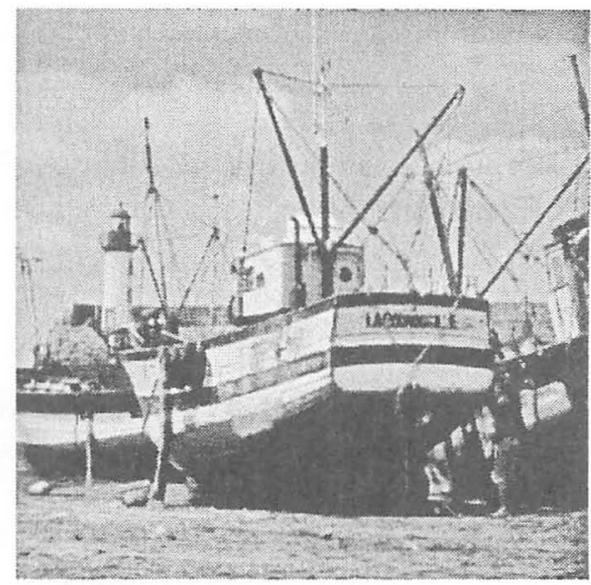

Fig. 5: Restored Image with Nonlinear Smoothing

Table 1: Mean Square Error

\begin{tabular}{|c|c|c|c|}
\hline Standard Images & Size & $M S E_{b s}$ & $M S E_{a s}$ \\
\hline \hline Boat & $512 \times 512$ & 0.1545 & 0.1311 \\
\hline Girl & $512 \times 512$ & 0.0237 & $4.8997 .10^{-4}$ \\
\hline Lenna & $256 \times 256$ & 0.0244 & 0.0017 \\
\hline Cameraman & $256 \times 256$ & 0.0234 & 0.0033 \\
\hline Goldhill & $256 \times 256$ & 0.02 & 0.0025 \\
\hline
\end{tabular}

\section{Conclusions}

The Bresenham line algorithm is effective to simulate the circular motion blurred image since it only involves incremental integer calculations and does not need the geometrical coordination transformation. The empty pixels problem which also happens in the logpolar sampling and the gemotrical coordinate transformation technique also happens in the use of Bresenham line algorithm. However, the position of the empty pixels can be easily known.

By decomposing circular motion blurring process as a series of blurring process along the circular path, the spatially variant circular image restoration problem can be reduced to a spatially invariant one. For the given center of rotation and the angle of rotation, the blurred image pixels along the circular blurring path can be calculated by using the Bresenham line algorithm. Then, the blurred image can be restored by applying inverse filtering to each circular path. Some unrestored image pixels that appear in the result of inverse filtering is caused by the fact that some pixels can be fetched along the circular path by the Bresenham line algorithm. Nonlinear smoothing proves to be effective to solve the problem. The use of median filter in image 


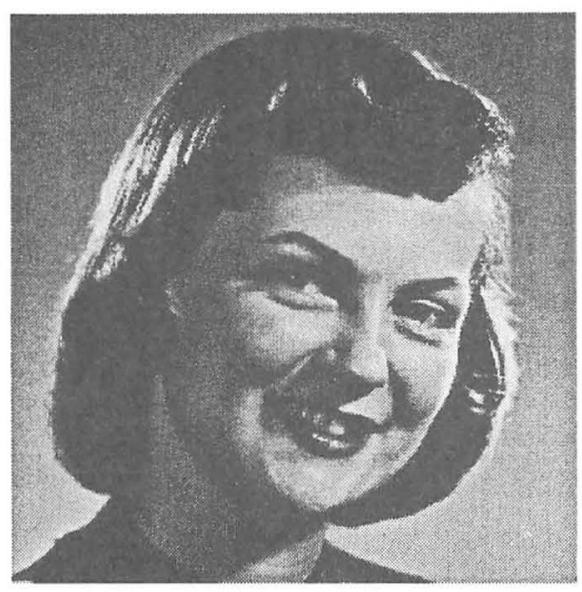

Fig. 6: Original Image

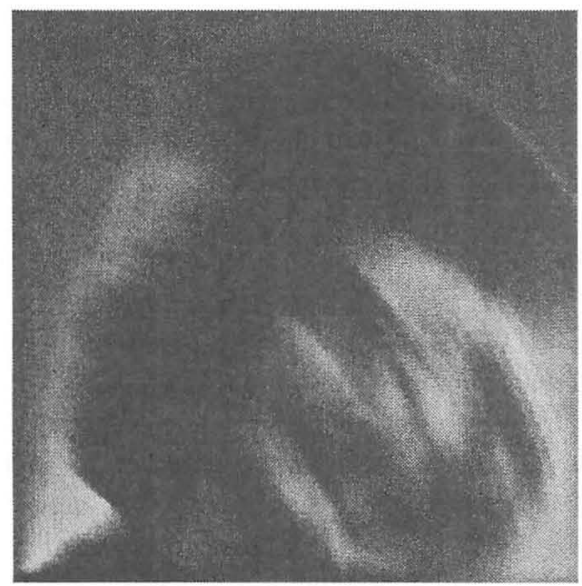

Fig. 7: Circular Motion Blurred Image

smoothing can keep the image structures intact.

\section{References}

[1] J. G. Nagy and D. P. O Leary: Restoring Images Degraded by Spatially Variant Blur, SIAM Journal on Scientific Computing, 19(4), pp.1063-1082, 1998.

[2] H. J. Trussel and S. Fogel: Identification and Restoration of Spatially Variant Motion Blurs in Sequential Images, IEEE Transactions on Image Processing, 1(1), pp.123-126, 1992.

[3] M. Sorrel and J. Flusser: Restoration of Color Images Degraded by Space-Variant Motion Blur, Springer-Verlag Berlin Heidelberg, pp. 450-457, 2007.

[4] M. Soumekh: Multiresolution Dynamic Image Representation with Uniform and Foveal Spiral

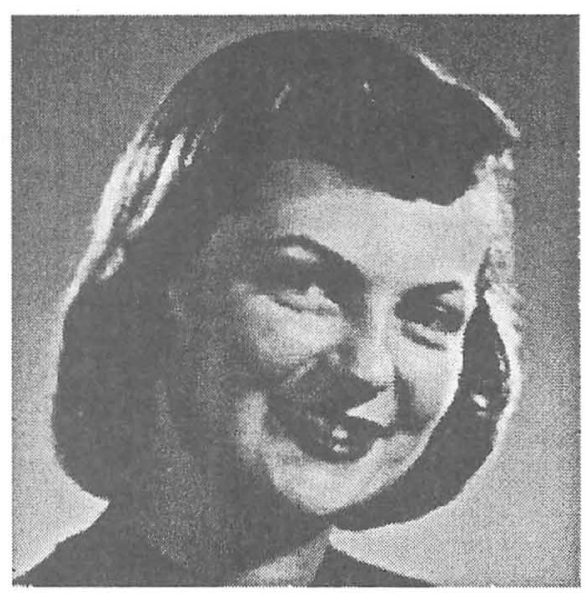

Fig. 8: Restored Image

Scan Data, IEEE Transactions on Image Processing, vol.7, no.11, pp.1627-1635, 1998.

[5] R. Wodnicki, G. Roberts, and M. Levine: A LogPolar Image Sensor Fabricated in a Standard 1.2- $\mu$ ASIC CMOS process, IEEE Journal of Solid-State Circuits, vol.32, no.8, pp.1274-1277, 1997.

[6] G. Bonmassar and E. Schwartz: Real-Time Restoration of Images Degraded by Uniform Motion Blur in Foveal Active Vision Systems, IEEE Transactions in Image Processing, vol.8, no.12, pp.1838-1842, 1999.

[7] D. Young: Straight Lines and Circles in the LogPolar Image, in M. Mirmehdi \&3 B. Thomas (Eds.), BMVC2000: Proceedings of the 11th British Machine Vision Conference, 11-14 September 2000, The University of Bristol, pp.426-435, 2000.

[8] N. Ono and R. Takiyama: On a Method for Polar Coordinate Transformation of Digital Images, Trans. IEICE,Japan, vol.J7-a, pp.75-83, January 1988.

[9] R. Takiyama: Image Restoration Considering the Image Quality, Proc. Conf. on Electronics and Electric Eng., Kyushu, September 1986.

[10] R. M. Hord and C. Sheffield: The Polar-Cartesian Picture Transform, Tech. Rep., Washington DC: Earth Satellite Co., 1975.

[11] D. Hearn and M. P. Baker: Computer Graphics, Prentice Hall, Englewood Cliffs, New Jersey, 1997.

[12] W. Burger and M. J. Burge: Digital Image Processing, Springer, New York, 2008.

[13] W. F. Al Maki, T. Shimahashi, T. Kitagawa, and S. Sugimoto: A New Approach of Blind Image Deconvolution Method without Artifact along the Blurring Paths, Proc. 39th ISCIE International Symposium on Stochastic Systems Theory and Its Application, 2007. 